\title{
ANALYTICAL STUDY OF THE QUADRATIC NONLINEAR OSCILLATOR BY JACOBIN METHOD AND COMPARISON WITH NHBM METHOD
}

\author{
Amin Moslemi Petrudi ${ }^{1}$ and Masoud Rahmani \\ Department of Mechanical Engineering, Tehran University, Tehran, Iran. \\ Corresponding author Email: \{1amin.moslemi2020@gmail.com\}
}

\begin{abstract}
The Jacobi method is a generalization of the simplex method for solving linear problems, or in other words, the Jacobi method is in a special case the same as the simplex method. One of the factors causing nonlinear effects in oscillating systems is the nonlinearity of the return force function, a wide range of which is modeled with the third-degree polynomial function and the selection of appropriate coefficients. In this paper, the Quadratic Nonlinear Oscillator using Jacobin elliptical sinus function is presented and compared with Newton Harmonic Balance Method. The method (NHBM) is done with Maple software and a comparison between the results of this method with the solution methods used by other researchers is shown to be a good match and can also be used for educational purposes.
\end{abstract}

Keywords: NHBM Method, Jacobin Method, Quadratic Nonlinear, Maple software.

\section{Introduction}

In 2004, Mains studied the quadratic solution of nonlinear oscillators. Quadratic nonlinear oscillators provided useful models both for testing turbulence methods and for analyzing various phenomena in physics and engineering. Different analytical methods are used for nonlinear equations that have recently received more attention. Among the researches that have been done so far in this field, we can mention the work of $\mathrm{HH}$, which has provided a real solution for the second-order nonlinear equation [1]. The approximate solution of the periods of the Helmholtz-Duffing equation, which is also used for the eardrum, has been investigated by Askari et al. [2]. JiHuan He first introduced the Homotopy perturbation method (HPM) in 1998. [3] Classic has an advantage, and the initial conjecture can be freely approximated by unknown constants, which can be determined by many methods, and the approximations obtained from this method are valid not only for small values of the Homotopy perturbation parameter but also for large values. In 2000, he used the Homotopy perturbation method for a series of nonlinear vibrational equations, including equations with different damping factors, as well as a sample of the singularity equation, and listed the advantages of this method over the classical Homotopy perturbation method. He used this method for the quantitative analysis of discrete nonlinear oscillators [4]. In 2005, with the help of his colleagues, he used this method to analyze a new nonlinear dynamic model of the spinning machine [5] and in his research, the optimal angle of convergence. Gain two strands of yarn in the $90^{\circ}$ equilibrium position and the resonance at 270 degrees. In 2005, the Homotopy perturbation method was used to solve the Differential Equations with complex numbers with nonlinear order of the third-degree type [6]. This paper presents the Jacobin elliptical sinus function and compared it with the Newton Harmonic Balance Method (NHBM).

\section{Equation of motion}

In general, quadratic nonlinear [8,7]:

$$
\begin{aligned}
& \ddot{x}+x+a x^{2}+\beta x \dot{x}+y(\dot{x})^{2}=0 \\
& \ddot{x}+x+\varepsilon x^{2}=0 . x(0)=A>0 . \dot{x}(0)=0
\end{aligned}
$$

Which is used as a mathematical model of human eardrum oscillation. [9] In general, nonlinear problems and most techniques are approximate and resort to several approximate solutions to the equation. [10] They include LP solution and improve The solution is in the harmonic balance method and the solution is in the iterative method. Therefore, Equation (2) can have an exact solution that provided the exact solution process for the equation [11]:

$$
x=a_{1}+a_{2} s n^{2}
$$

Where $s n=s n(\omega t+b . m))$ is the function of the Jacobin elliptical sinus and $\left(a_{1}, a_{2}, \omega . b . m\right)$ are its constants. Therefore, $\mathrm{m}$ and $s n$, which can be expressed as the constants $\left(a_{1} \cdot a_{2}, \omega\right)$ as Equation (4): 


$$
\begin{gathered}
a_{1}=\frac{1+m-\sqrt{\lambda}}{2 \sqrt{\lambda}} \cdot a_{2}=-\frac{3 m}{2 \sqrt{\lambda}} \cdot \omega=\frac{1}{2 \lambda^{1 / 4}} \\
\lambda=m^{2}-m+1
\end{gathered}
$$

\section{Problem-solving method}

Using the initial conditions in Equations (2) and (3):

$$
x(t)=A+a_{2} s n^{2}=A+a s n^{2}(\omega t . m)
$$

That the first and second-order derivatives in Equation (6) are equal to:

$$
\begin{aligned}
& \dot{\mathrm{x}}=2 a \omega s n(\omega t . m) c n(\omega t . m) d n(\omega t . m) \\
& \ddot{x}=2 a \omega^{2}\left[1-2(1+m) s n^{2}+3 m s n^{4}\right]
\end{aligned}
$$

In Equation (7), $c n$ and $d n$ are cosmic and of the third-order function type in the Jacobin method, which replaces Equation (6) and (8) of Equation (2), respectively:

$$
\begin{aligned}
& 2 a \omega^{2}+A+\varepsilon A^{2}+\left[a+2 A a \varepsilon-4(1+m) a \omega^{2}\right] s n^{2} \\
& +\left(6 m a \omega^{2}+\varepsilon a^{2}\right) s n^{4}=0
\end{aligned}
$$

Determining the coefficients $s n^{0}, s n^{2}, s n^{4}$ leads to zero and becomes the following algebraic equations:

$$
\begin{aligned}
& 2 a \omega^{2}+A+\varepsilon A^{2}=0 \\
& a+2 A a \varepsilon-4(1+m) a \omega^{2}=0 \\
& 6 m a \omega^{2}+\varepsilon a^{2}=0
\end{aligned}
$$

From Equation (12):

$$
a=\frac{-6 m \omega^{2}}{\varepsilon}
$$

By substituting in Equation (10):

$$
12 m \omega^{4}-\varepsilon A(1+\varepsilon A)=0
$$

From Equation (11):

$$
\omega^{2}=\frac{1+2 \varepsilon A}{4(1+m)}
$$

By substituting Equation (15) for (14):

$$
\begin{aligned}
& 4 p(1+p) m^{2}-[3+4 p(1+p)] m \\
& +4 p(1+p)=0 . p=\varepsilon A
\end{aligned}
$$

To solve m:

$$
=\frac{m_{1.2}}{3+4 p(1+p) \pm \sqrt{9+24 p\left(1-p-4 p^{2}-2 p^{3}\right)}}
$$

If $\varepsilon \rightarrow 0(p \rightarrow 0)$ then $m=0$ Therefore:

$$
\begin{aligned}
& m(p) \\
& =\frac{1}{2}+\frac{3-(1+2 p) \sqrt{3(1-2 p)(3+2 p)}}{8 p(1+p)} \\
& =\frac{1}{2}+\frac{3\left(2 p^{2}+2 p-1\right)}{3+(1+2 p) \sqrt{3(1-2 p)(3+2 p)}}
\end{aligned}
$$

By substituting Equation (18) into Equation (15) and simplifying the result:

$$
\omega(p)=\sqrt{1+\frac{8 P}{3 \pi}}
$$

By substituting Equation (18) and Equation (19) in Equation (13):

$$
\begin{aligned}
& a(\varepsilon \cdot A) \\
& =[\sqrt{3(1-2 p)(3+2 p)-3(1+2 p)}] / 4 \varepsilon \\
& =\frac{-12 p(1+p)}{\varepsilon[\sqrt{3(1-2 p)(3+2 p)-3(1+2 p)}]}
\end{aligned}
$$

\section{Results and Discussion}

Following Equation (18): $p \leq \frac{1}{2}$ but if $p=\frac{1}{2}$, in Equation (2) which is an equation that has a homoclinic circuit with period $\infty+$. Therefore, the solution of the period provided.

First, the results are compared with the Newton harmonic balance method [12] for the values of $\mathrm{A}=0.3$ and $\varepsilon=1$, which can be seen in Fig. 1 .

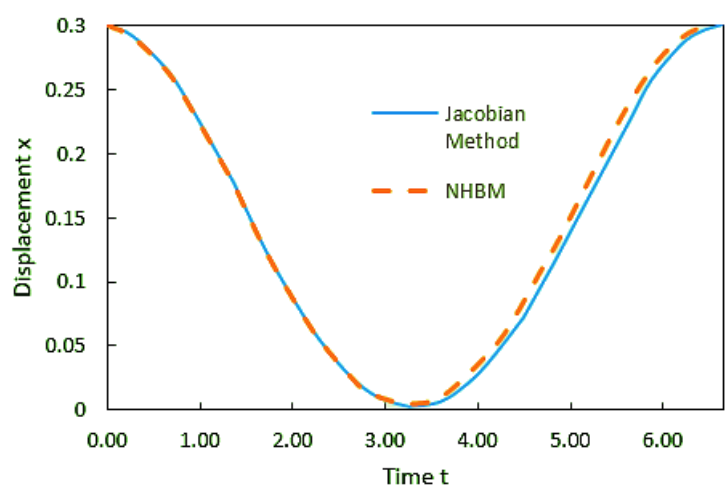

Fig. 1: Comparison of the Jacobin method with NHBM method. 
According to Fig. 1, the Jacobin method has the necessary accuracy. In the following, the various dimensions of the ability of this method to solve problems are investigated.

4.1. In special cases $\varepsilon=0$

$$
\begin{aligned}
& \text { If } \varepsilon=0 \text { in Equation (2) }[13,14]: \\
& \qquad \ddot{x}+x=0 \cdot x(0)=A>0 \dot{x}(0)=0
\end{aligned}
$$

By placing in Equation (18) - (20):

$$
\begin{aligned}
& m(0)=0 . \omega(0)=\frac{1}{2} \cdot a(0 \cdot A)= \\
& \lim \varepsilon \rightarrow 0 \quad a=-2 A
\end{aligned}
$$

According to Equation (6):

$$
\begin{aligned}
& x(t)=A-2 A \operatorname{sn}^{2}\left(\frac{t}{2} \cdot 0\right) \\
& =A-2 A \sin ^{2}\left(\frac{t}{2}\right)=A \cos t
\end{aligned}
$$

Which leads to the solution of Equation (21).

\subsection{Domain B for $x \leq 0$}

Assuming that the system oscillates between asymmetric ranges $[-B \cdot A](B>0)$, according to Equation (6):

$$
-B=A+a
$$

By substituting in Equation (20) in (24):

$$
\begin{aligned}
& B(\varepsilon . A)=\frac{3+2 p-\sqrt{3(1-2 p)(3+2 p)}}{4 \varepsilon} \\
& =\frac{1}{4}\left[3+2 \varepsilon A-3 \sqrt{1-\frac{4}{3}} \varepsilon A(1+\varepsilon A)\right]
\end{aligned}
$$

Specifically,

$$
B(0 . A) \lim \varepsilon \rightarrow 0 \quad B=A . B\left(\frac{0.5}{A} \cdot A\right)=2 A
$$

By placing in Equation (25):

$$
\begin{aligned}
& B(\varepsilon . A) \\
& =\frac{\sqrt{3+2 p}[\sqrt{3+2 p-\sqrt{3(1-2 p)}}}{4 \varepsilon} \\
& =\frac{2 p \sqrt{3+2 p}}{\varepsilon[\sqrt{3+2 p+\sqrt{3(1-2 p)}}}
\end{aligned}
$$

It is easily shown that:

$$
\sqrt{3+2 p} \geq \sqrt{3(1-2 p)} . \quad 0 \leq p \leq 0.5
$$

Thus:

$$
\begin{aligned}
& A=\frac{2 p \sqrt{3+2 p}}{\varepsilon[\sqrt{3+2 p}+\sqrt{3+2 p]}} \leq B(\varepsilon . A) \\
& \leq \frac{2 p \sqrt{3+2 p}}{\varepsilon \sqrt{3+2 p}}=2 A
\end{aligned}
$$

Obviously if 0.5 and $p \neq 0$

$$
A<B(\varepsilon . A)<2 A
$$

Equation (25) is solved using the algebraic equation:

$$
\frac{B^{2}}{2}-\frac{\varepsilon B^{3}}{3}=\frac{A^{2}}{2}+\frac{\varepsilon A^{3}}{3}
$$

\subsection{Exact period of oscillation $\mathrm{T}$ :}

Using the relation $\operatorname{sn}(u \pm 2 F)=-\operatorname{sn}(u)[17,16,15]$

$$
s n^{2}(u \pm 2 F)=s n^{2}(u)
$$

$$
F=F\left(k \cdot \frac{\pi}{2}\right)=\int_{0}^{\pi / 2} \frac{d \theta}{\sqrt{1-k^{2} \sin ^{2} \theta}} \cdot k^{2}=m
$$

$\mathrm{T}$ specifies the exact time of the oscillation. Then using equation (6):

$$
a s n^{2}(\omega t \pm \omega T . m)=a s n^{2}(\omega t . m)
$$

Comparing Equation (34) with (32):

$$
T(p)=\frac{2 F}{\omega}
$$

Fig. 2 shows the difference of $m$ with relative to $p$, Fig. 3 shows the difference of $\omega$ with relative to p, and Fig. 3 shows the difference of a with relative to $\varepsilon$ for $\mathrm{A}=1$. 


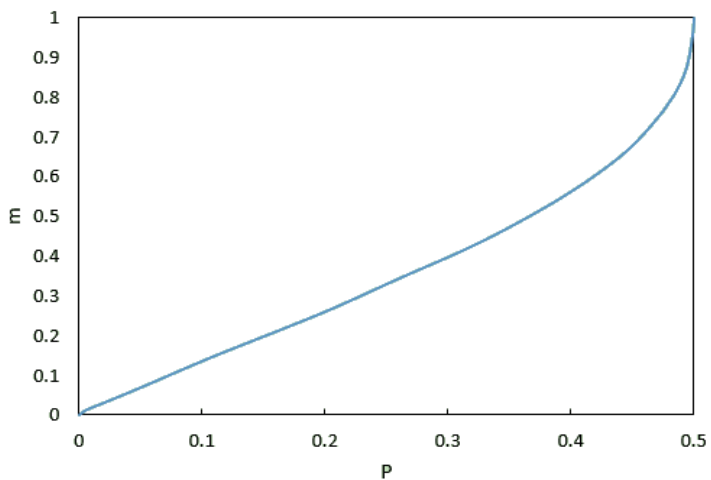

Fig. 2. The difference between $\mathrm{m}$ and $\mathrm{p}$.

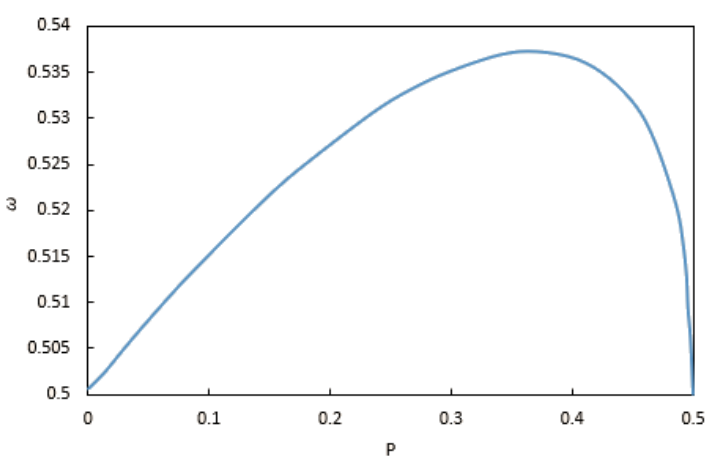

Fig. 3. Difference $\omega$ with relative to $\mathrm{p}$.

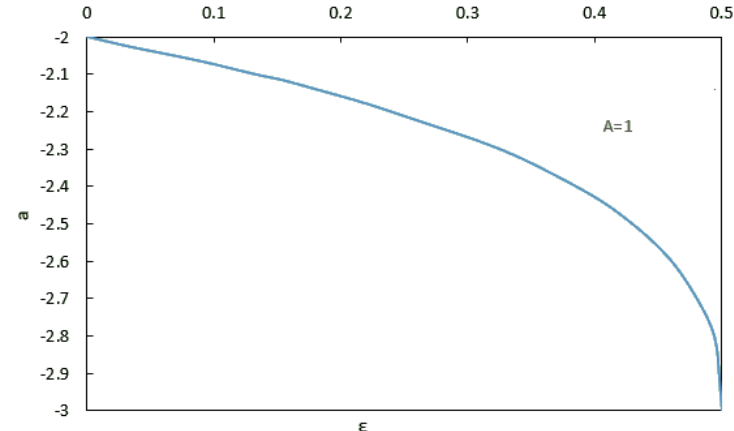

Fig. 4. The difference between an and $\varepsilon$ for $A=1$.

For two special cases:

$$
T(0)=2 \pi . \quad T(0.5)=+\infty
$$

Fig. 4 shows the difference $B$ with relative to $\varepsilon$ for $A=1$ and Fig. 5 shows the difference of $T$ with relative to p. Fig. 6 shows the displacement overtime for some time $x(t)$ relative to $\mathrm{A}=1$ and $\varepsilon=0.45$.

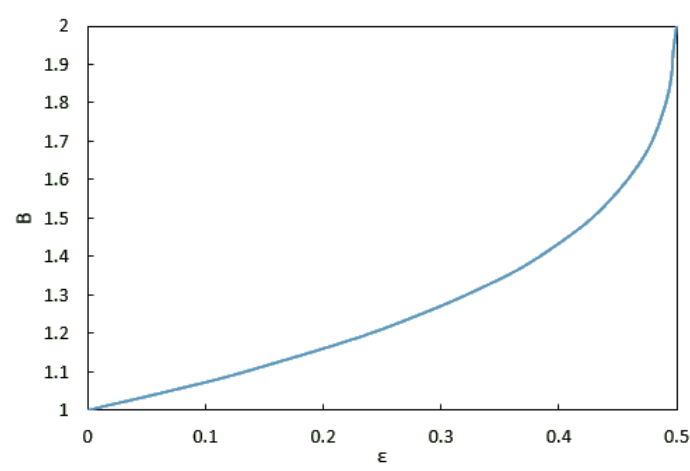

Fig. 4. The difference between $\mathrm{B}$ and $\varepsilon$ for $\mathrm{A}=1$.

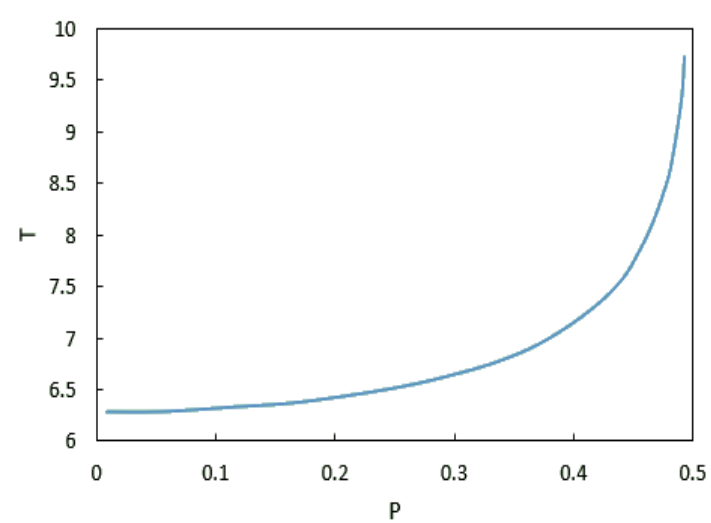

Fig. 5. The difference of $\mathrm{T}$ with relative to $\mathrm{p}$.

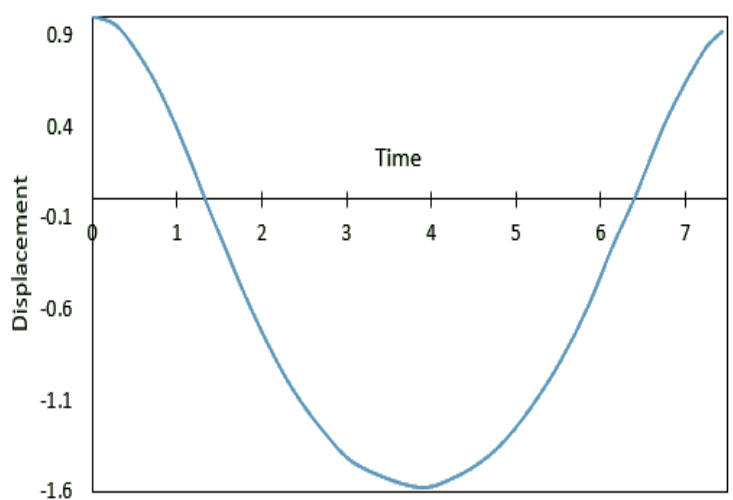

Fig. 6. Displacement over time.

Using the Duffing equation:

$$
\ddot{x}+\dot{x}+\varepsilon x^{3}=0 . \quad x(0)=A . \quad \dot{x}(0)=0
$$

By comparison in Equation (37), it can be written that:

$$
x(t)=\operatorname{Acn}(\omega t . m)
$$

Than:

$$
\omega=\sqrt{1+\varepsilon A^{2}} . \quad m=k^{2}=\frac{\varepsilon A^{2}}{2\left(1+A^{2}\right)}
$$

The joining of the period of precise fluctuations is expressed as follows:

$$
T=\frac{4 F}{\omega}=\frac{4 F}{\sqrt{1+\varepsilon A^{2}}}
$$

The solution of the quadratic nonlinear oscillator in Equation (2) and the nonlinear oscillator in Equation (37) is expressed by the Jacobin elliptic functions. Therefore, in the following, the short equation of differential equations is considered:

$$
\ddot{x}+x+\alpha x^{2}+\beta x^{3}=0 \cdot x(0)=A \cdot \dot{x}(0)=0
$$




\section{Conclusion}

The exact solution in Equation (6) is modeled and proposed using the QNO quadratic nonlinear oscillator relative to Equation (2). The presented in this article can be used as a criterion for solving various approximate problems and can also be used for educational purposes. Has been. And the comparison between the results of this method is done with the solution methods used by other researchers, which shows a good agreement.

\section{References}

[1] Hu, H. (2006). Exact solution of a quadratic nonlinear oscillator. Journal of sound and vibration, 450-457.

[2] Askari, H., Saadatnia, Z., Younesian, D., Yildirim, A., \& Kalami-Yazdi, M. (2011). Approximate periodic solutions for the Helmholtz-Duffing equation. Computers \& Mathematics with Applications, 62(10), 3894-3901.

[3] Wu, B.S., Sun, W.P., Lim, C.W., "An analytical approximate technique for a class of strongly nonlinearoscillators", International Journal of Non-Linear Mechanics, Vol. 41, pp. 766-774, 2006

[4] MohammadMehdi Mashinchi Joubari, Hamid Javanian Jouybari , Mohammad Hadi Pashaei., "Solution of strongly nonlinear oscillator problem arising in Plasma Physics with Newton Harmonic Balance Method", Journal of Applied and Computational Mechanics, Vol. 1, No. 2, pp. 59-66, 2015.

[5] 1M. Ghadimi ,H.D. Kaliji., "Application of the Harmonic Balance Method on Nonlinear Equations", World Applied Sciences Journal 22 (4),pp. 532-537, 2013.

[6] Oyedeji, F. O., Erazua, E. A., \& Adeleke, B. B. (2018). GC-Mass Spectroscopic Chemical Characterization and Physico-Chemical Properties of Oil From Seed Kernels of Four Cultivars of Magnifera Indica. European Journal of Pure and Applied Chemistry Vol, 5(1).
[7] Mickens, R. E. (2004). Quadratic non-linear oscillators. Journal of sound and vibration, 1(270), 427-432.

[8] Mickens, R. E. (1996). Oscillations in planar dynamic systems (Vol. 37). World Scientific.

[9] Wu, B. S., \& Lim, C. W. (2004). Large amplitude non-linear oscillations of a general conservative system. International Journal of Non-Linear Mechanics, 39(5), 859-870.

[10] Li, P. S., \& Wu, B. S. (2004). An iteration approach to nonlinear oscillations of conservative singledegree-of-freedom systems. Acta Mechanical, 170(12), 69-75.

[11] Rand, R. H. (1992). Using computer algebra to handle elliptic functions in the method of averaging. ASMEPUBLICATIONS-PVP, 205, 311-311.

[12] Rahmani, Masoud, Ionut Cristian Scurtu, and Amin Moslemi Petrudi. "Analytical and Dynamic study of Pulled Mass Nonlinear Vibration by Two Cables using Newton's Harmonic Balance Method." Technium: Romanian Journal of Applied Sciences and Technology 2.2 (2020): 79-86.

[13] Hu, H. (2006). Solution of a quadratic nonlinear oscillator by the method of harmonic balance. Journal of Sound and Vibration, 293(1-2), 462-468.

[14] Mickens, R. E. (Ed.). (2004). Mathematical methods for the natural and engineering sciences.

[15] Thomson, W. (2018). Theory of vibration with applications. CrC Press.

[16] Yazdi, M. K. (2018). An accurate relationship between frequency and amplitude to nonlinear oscillations. Journal of Taibah University for Science, 12(5), 532-535.

[17] Shevtsov, B., \& Shevtsova, O. (2018). Fluctuations and nonlinear oscillations in complex natural systems. In E3S Web of Conferences (Vol. 62, p. 02006). 